the patients with meningitis. It is of interest that in 3 of the bacteriologically confirmed cases of meningitis the counter-current immunoelectrophoresis for antigen was negative, yet a positive result to the Limulus test was obtained; this reaffirms the suggestion of Berman et al. ${ }^{8}$ that the limulus test should be included in the initial assessment of patients who may have early or partially treated Gram-negative meningitis.

The reliability of detecting endotoxin in the CSF of Gram-negative meningitis shown by Berman et al. $^{8}$ does not, however, obtain in the plasma of meningococcaemic patients, or patients with other Gram-negative bacteraemias. ${ }^{5}$

\footnotetext{
References

1 Whittle H C, Greenwood B M, Davidson N McD, et al. Meningococcal antigen in diagnosis and treatment of group A meningococcal infections. Am J Med 1975; 58: 823-8.

2 Lewis L S. Prognostic factors in acute meningococcaemia. Arch Dis Child 1979; 54: 44-8.
}

${ }^{3}$ Levin J, Tomasulo P A, Oser R S. Detection of endotoxin in human blood and demonstration of an inhibitor. $J$ Lab Clin Med 1970; 75: 903-11.

4 Greenwood B M, Whittle H, Dominic-Rajkovic O. Counter-current immuno-electrophoresis in the diagnosis of meningococcal infection. Lancet 1971; ii: 519-21.

5 Levin J, Poore T E, Young N S, et al. Gram-negative sepsis: detection of endotoxemia with limulus lysate test with studies of associated changes in blood coagulation, serum lipids, and complement. Ann Intern Med 1972; 76: 1-7.

${ }^{6}$ Chien S, Chang C, Dellenback R J, Usami S, Gregersen M I. Hemodynamic changes in endotoxin shock. Am J Physiol 1966; 210: 1401-10.

7 Muller-Berghaus G, Lohmann E. The role of complement in endotoxin induced disseminated intravascular coagulation. Br J Haematol 1974; 28: 403-18.

8 Berman N S, Siegel S E, Nachum R, Lipsey A, Leedom J. Cerebrospinal fluid endotoxin concentrations in Gramnegative bacterial infections. J Pediatr 1976; 88: 553-6.

Correspondence to Dr H R Tubbs, Infectious Disease Unit, North Staffordshire Hospital Centre, Bucknall Hospital, Bucknall, Stoke-on-Trent ST2 8LD.

\title{
D-lactic acidosis in a boy with short bowel syndrome
}

\author{
E P SCHOOREL, M A H GIESBERTS, W BLOM, AND H H VAN GELDEREN \\ Department of Paediatrics, University Hospital, Leiden, and Sophia Children's Hospital, \\ Erasmus University, Rotterdam, The Netherlands
}

SUMMARY Metabolic acidosis in a 3-year-old child with short bowel syndrome led to the discovery of massive D-lactic aciduria. After normalisation of the intestinal bacterial flora, D-lactate disappeared together with the acidosis. Dysbacteriosis with excessive production of D-lactate by intestinal bacteria (unidentified) and subsequent absorption explains this unusual cause of metabolic acidosis.

L-lactic acidaemia is a well-known cause of acidosis in childhood. D-lactic acid is not normally present in the urine of humans and can easily be overlooked in cases of acidosis.

We report the case of a child with short bowel syndrome in whom acidosis with an increased anion gap led us to the finding of D-lactic aciduria.

\section{Case report}

The patient, a boy born on 8 December 1975, was first seen in the cardiological division of our department at age 3 days because of transposition of the great vessels; a Rashkind septostomy was performed, later followed by a Blalock anastomosis and, at age 20 months, by total correction.

At age 10 months thrombosis of the mesenteric vessels occurred during an attack of gastroenteritis with dehydration. Resection of $140 \mathrm{~cm}$ of the smallbowel, the caecum, and $3 \mathrm{~cm}$ of ascending colon was necessary. The resulting malabsorption was treated with dietary measures, including medium-chain triglycerides, restriction of disaccharides and, for a period of 9 months, cholestyramine. This treatment was supervised by the local paediatrician and had only partial success: height and weight increases were below normal.

At age 38 months the boy was readmitted to our department because of attacks of 'dyspnoea' and drowsiness. His mother reported that he had had such attacks occasionally since the intestinal resection but that these had increased to an almost daily frequency in the last weeks. On such days he seemed hungry, unhappy, weak, and uncertain in moving; subsequently he started to breathe deeply and became drowsy. This persisted for a few hours and subsided gradually. At no time was he comatose and he did not convulse. Increasing the 
frequency of meals had no influence on the symptoms.

Similar attacks were noted after admission. Blood glucose levels were always normal. Further examination showed pronounced signs of malabsorption including growth (height increase only $5.5 \mathrm{~cm}$ during the year), reduced fat absorption ( $71 \%$ ), low blood levels of vitamin D and B12 and iron, reduced hydroxy-proline excretion, pronounced osteoporosis (skeletal index 0.03952), and a bone age of only 9 months at the wrist. Serum electrolytes showed an 'anion gap': sodium 143, potassium $4 \cdot 6$, chloride 104 , and bicarbonate $16 \cdot 4 \mathrm{mmol} / \mathrm{l}$. Repeated blood examination showed varying degrees of metabolic acidosis, lowest $\mathrm{pH} \mathbf{7 \cdot 2 3}$ and lowest base excess $-17 \mathrm{mmol} / \mathrm{l}$. There was no ketosis. Serum L-lactic acid was normal. Faecal pH was only $4 \cdot 3$. Analysis of organic acids in the urine was performed by gasliquid chromatography and mass spectrometry. A massive lactic acid excretion was detected. As the excretion of L-lactic acid in the urine already had been found to be only slightly increased* and no pyruvic acid was present in the urine, it seemed likely that the lactic acid was D-lactic acid. A high excretion of phenyl lactic acid and 4-OH-phenylacetic acid in the urine was also noted, indicating abnormal phenylalanine and tyrosine absorption. This, together with the signs of severe malabsorption mentioned earlier, strongly suggested that the D-lactate was produced by unusual bacteria in the gut. We subsequently followed the urinary D-lactate excretion using a direct enzymatic method.*

Bacteriological analysis of the faecal flora showed a poorly mixed anaerobic flora with predominance of Gram-positive and too few Gram-negative bacteria.

In the meantime acidosis had been corrected by sodium bicarbonate given orally. Although this prevented further attacks, D-lactate-aciduria continued. For 5 consecutive days we gave a standard bacterial flora called 'Julia flora' $\dagger$ This flora has been used for recolonisation after intestinal decontamination-for example, in patients treated with bone marrow transplantation. ${ }^{1}$ In our case no decontamination of the gut was performed before the Julia flora was given.

After this recolonisation the faecal flora consisted of predominantly Gram-negative bacteria. Dlactate excretion fell rapidly after this change of intestinal bacterial composition (Table) and had virtually disappeared 11 days later. Bicarbonate administration could be stopped, acidosis did not

\footnotetext{
*Boehringer L-lactic acid test, Cat No 139084 , D(-) LDH, Cat No 106941, and D $(-)$ lactate Cat No 106917 was used.

$†$ Provided by TNO, Delft.
}

Table D-lactate excretion ( $\mathrm{mmol} / 24 \mathrm{~h})$

\begin{tabular}{ll}
\hline Before gut recolonisation & $20-36$ \\
After colonisation & 0.50 \\
4 days & 0.015 \\
\hline 11 days & \\
\hline
\end{tabular}

recur, and there have been no attacks in the 9 months since discharge.

\section{Discussion}

D-lactic acid is normally not produced by humans. In ruminants acidosis caused by accumulation of absorbed D-lactic acid from the rumen is a wellknown result of overfeeding with cereals or other readily fermentable carbohydrates. ${ }^{2}$ In our patient an increased anion gap made us consider organic acidaemia. Urine analysis for organic acids, which is routine in such circumstances, led to the diagnosis. Although we did not measure the blood levels of D-lactate there is little doubt that the acidotic attacks were due to this acid which cannot be metabolised in humans.

We suggest that the following series of events occurred: a relatively large amount of carbohydrates reached the colon leading to a lowering of the local pH. In such circumstances there could be an abundant overgrowth of unusual bacteria - for example, the 'lactic acid bacteria'. Some species of this group can produce not only L-lactic acid but also Dlactic acid.

The restoration of normal intestinal flora and the correction of intestinal $\mathrm{pH}$ would then explain the rapid disappearance of D-lactaturia, although it was not feasible to identify the species which produced this acid. No chemotherapeutic decontamination of the bowel was used, but the gut was recolonised with normal faecal flora. No special dietary measures were taken.

Recently a similar case of D-lactate acidosis was described in a 30-year-old man with short bowel syndrome. $^{3}$ The assumed dysbacteriosis was treated successfully with neomycin. We know of only one other report of D-lactic aciduria ${ }^{4}$ and this was in a mentally retarded child. However, the amount of D-lactate excretion was very much lower than in our patient and no acidosis was present. The child did not have intestinal disease and a bacterial origin of the D-lactate could not be proved.

\section{References}

1 van der Waaij D, Vossen J M, Korthals Altes C, Hartgrink C. Reconventionalization following antibiotic decontamination in man and animals. Am J Clin Nutr 1977; 30: 1887-95. 
2 Dunlop R H, Hammond P B. D-lactic acidosis of ruminants. Ann NY Acad Sci 1965; 119: 1109-32.

${ }^{3}$ Oh M S, Phelps K R, Traube M, Barbosa-Saldivar J L, Boxhill C, Carroll H. D-lactic acidosis in a man with the short-bowel syndrome. $N$ Engl J Med 1979; 301 : 249-52.

4 Duran M, van Biervliet J P G M, Kamerling J P, Wad- man S K. D-lactic aciduria; an inborn error of metabolism? Clin Chim Acta 1977; 74: 297-300.

Correspondence to Professor Dr H H van Gelderen, Department of Paediatrics, University Hospital, Leiden, The Netherlands.

\title{
Hypoproteinaemia, oedema, and anaemia: an unusual presentation of cystic fibrosis in dizygotic twins
}

\author{
I BLUMENTHAL AND D W FIELDING \\ Department of Paediatrics, Chester City Hospital, Chester
}

SUMMARY The rare combination of oedema, hypoproteinaemia, and anaemia as a presenting feature of cystic fibrosis in dizygotic twins of opposite sex is described. The features of this syndrome together with pathogenesis, treatment, and prognosis are discussed.

Cystic fibrosis seldom affects both members of a dizygotic twin pair. This paper reports twins of opposite sex with the unusual presentation of hypoproteinaemia, anaemia, and oedema.

\section{Case report}

Dizygotic twins were born by vaginal delivery to a 29-year-old white primigravida after an uneventful pregnancy and labour. Their estimated gestational age was 37 weeks. The first twin was a boy weighing $2.46 \mathrm{~kg}$, and the second a girl weighing $1.98 \mathrm{~kg}$. Both were breast fed with occasional supplements of cows' milk (SMA Gold Cap). Weight gain was slow despite voracious appetites and satisfactory calorie intakes. Screening tests to exclude infection and metabolic errors showed a urinary infection in twin 1 . Treatment produced no improvement. The twins were discharged at 4 weeks; it had been noted that they cried excessively and did not settle after feeds. At discharge their respective weights were 2.52 and $2.38 \mathrm{~kg}$. Assessment at 7 weeks showed minimal weight gain (Figure); physical examination was normal.

At 9 weeks admission was precipitated by the development of anasarca and pallor. On examination each twin was pale and had generalised pitting oedema. With the exception of slight jaundice no other abnormality was noted. Blood pressures were normal. There was no proteinuria. Investigations showed anaemia and hypoproteinaemia; both twins were transfused. The results of investi- gations are shown in the Table. The consistent increase in sweat chloride concentration together with absent faecal trypsin led to the diagnosis of cystic fibrosis. Chest $x$-rays were normal. Chromosome analysis was $46 \mathrm{XY}$ (twin 1) and $46 \mathrm{XX}$ (twin 2).

13 weeks after birth treatment with pancreatic extract (Pancreatin BP) and flucloxacillin was begun. The formula (SMA) used to supplement breast feeding was replaced by a high protein
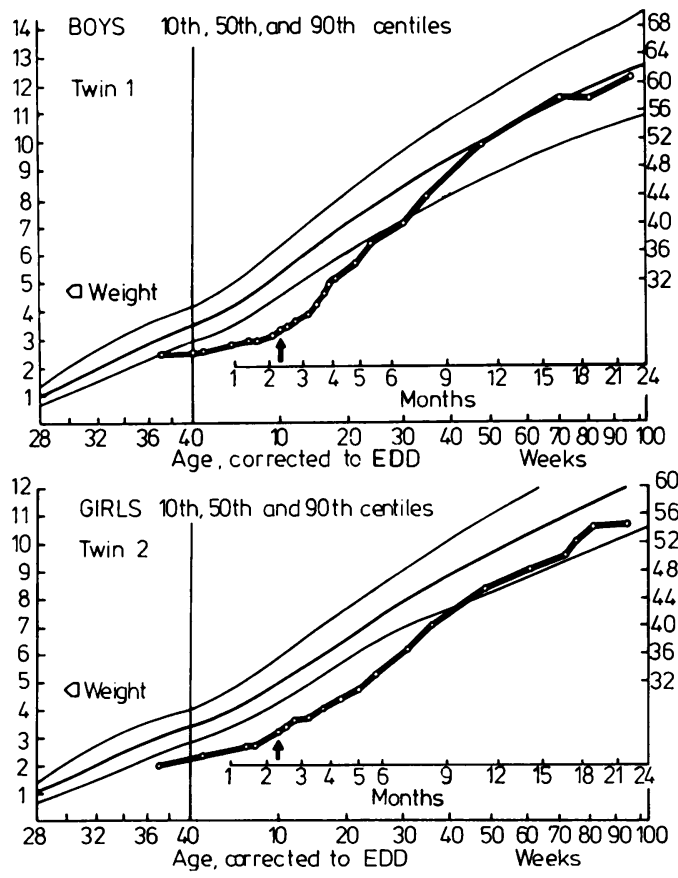

Figure Weights of twins plotted on growth charts. Arrows indicate start of pancreatic extract and high protein formula. 